\title{
A Novel Omnidirectional Mobile Robot With Wheels Connected by Passive Sliding Joints
}

\section{$\operatorname{AUTHOR}(\mathrm{S}):$}

Terakawa, Tatsuro; Komori, Masaharu; Matsuda, Kippei; Mikami, Shinji

\section{CITATION:}

Terakawa, Tatsuro ... [et al]. A Novel Omnidirectional Mobile Robot With Wheels

Connected by Passive Sliding Joints. IEEE/ASME Transactions on Mechatronics 2018, 23(4): 1716-1727

\section{ISSUE DATE:}

2018-08

URL:

http://hdl.handle.net/2433/241005

RIGHT: 


\title{
A Novel Omnidirectional Mobile Robot with Wheels Connected by Passive Sliding Joints
}

\author{
Tatsuro Terakawa, Masaharu Komori, Member, IEEE, Kippei Matsuda, and Shinji Mikami
}

\begin{abstract}
Mobile robots for automatically transporting products in factories and warehouses contribute to an increase in efficiency. Omnidirectional mobile robots can move immediately in an arbitrary direction and overcome the disadvantages of lack of mobility in conventional mobile robots. However, the omnidirectional mobile robots proposed in the past have not been as reliable as the conventional mobile robots such as AGVs due to their complicated wheel mechanisms. This research proposes a novel omnidirectional mobile robot named SWOM (slidable-wheeled omnidirectional mobile robot), which has three wheels that connect to the robot body by three passive sliding joints. The relative movements of the sliding joints allow SWOM to use conventional wheels. Thus, SWOM realizes both omnidirectional mobility and structural reliability. In this paper, we discuss the kinematic conditions for omnidirectional mobile robots and prove theoretically that SWOM can achieve omnidirectional movement. We present a kinematic analysis, a reachable region evaluation considering the limited movable range of the sliding joints, and trajectory generation that enables SWOM to move unlimitedly. We develop a prototype of SWOM and conduct experiments that show SWOM actually moves according to the theory. From the above, we verify the effectiveness of SWOM as an omnidirectional mobile robot.
\end{abstract}

Index Terms-Mobile robot kinematics, mobile robots, motion analysis.

\section{INTRODUCTION}

$\mathrm{M}$ OBILE robots such as AGVs are used to carry products in factories and warehouses for automation and efficiency. The typical mobile robot is either a differential wheeled robot, which has two parallel fixed wheels [Fig. 1(a)] that move independently, or a steerable wheeled robot, which has one or more orientable wheels [Fig. 1(b)] that can turn around the steering axis and change direction. These wheeled mobile robots have the advantage of a simple and reliable structure. However, they are nonholonomic mobile robots. This means that they cannot move immediately in certain directions. Therefore, they need a switchback or a turnabout when moving

Manuscript received February 15, 2017; revised July 31, 2017 and November 27, 2017; accepted May 17, 2018. Date of publication MMMM DD, YYYY; date of current version MMMM DD, YYYY. Recommended by Technical Editor XXXX. This work was supported by JSPS KAKENHI Grant Number JP18J13377.

The authors are with the Department of Mechanical Engineering and Science, Kyoto University Graduate School of Engineering, Kyoto 6158540, Japan (e-mail: komorim@me.kyoto-u.ac.jp).

Color versions of one or more of the figures in this paper are available online at http://ieeexplore.iee.org.

Digital Object Identifier XXXXXXXX in a particular direction, such as sideways. Such movements require extra time and space.

In contrast, omnidirectional mobile robots can move immediately in an arbitrary direction. This superb mobility makes a switchback or a turnabout unnecessary and enables the robot to move efficiently. A typical omnidirectional mobile robot has omni wheels [Fig. 1(c)] or Mecanum wheels [Fig. 1(d)], whose circumferences consist of free rollers [1]-[7]. Their mobility allows applications such as a variable wheel arrangement mechanism [4], a highly efficient control method [5], and a competition robot with an omni-vision system [6]. The active omni wheel [8] enables both the main body of the wheel and the rollers to rotate actively. However, the omni wheel has disadvantages such as a low load capacity due to its complicated structure, unavoidable vertical vibration due to discontinuity of the ground contact point, and inability to surmount high bumps due to the small radius of the free rollers [9]. As an alternative, the orthogonal wheel, which has two spherical-segment wheels, has been proposed [10]-[14], but the complicated structure and the discontinuous contact point remain. Spherical wheels can be used for omnidirectional mobile robots [15]-[20], but then size becomes a problem. Spherical wheels tend to be larger than other types of wheels, but downsizing the wheels makes it difficult for the robot to surmount high bumps.

To address the problems caused by the specialized structure of various wheels, omnidirectional mobile robots using caster wheels [Fig. 1(e)] have been proposed [21]-[29]. A caster wheel corresponds to an orientable wheel with an offset between the wheel axle and the steering axis, so that the wheel itself is a conventional wheel (for example, a tire). Therefore, the problems of a specialized structure do not occur in robots with caster wheels. However, caster wheels may turn too quickly and become unstable when reversing. In addition, if both the wheel axle and the steering axis are motorized, two or more caster wheels cause redundancy and so the motors have to drive with high accuracy. Non-redundant mechanisms have also been proposed [25], [26], but the design flexibility and the transmission path design pose problems.

To solve the problems with these mobile robots, we propose a novel mechanism for an omnidirectional mobile robot in this study. The robot has three orientable wheels connected to the main body by passive sliding joints. The orientable wheel, as well as the caster wheel, eliminates the problems of specialized wheels such as the omni wheel. Furthermore, unlike the caster wheel, the orientable wheel with a non-offset steering axis is never in the overconstraint-like situation, even when redundant 


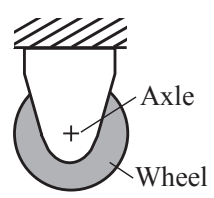

(a)

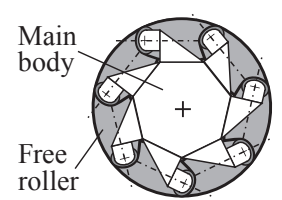

(d)

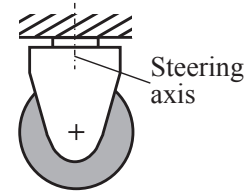

(b)

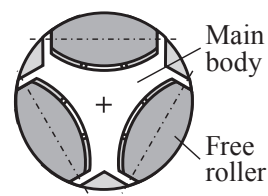

(c)

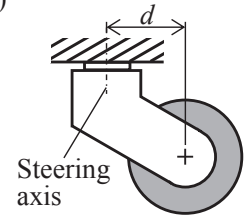

(e)
Fig. 1. Varieties of wheel mechanisms. (a) Fixed wheel, where only the wheel itself can rotate. (b) Orientable wheel, where the wheel can both rotate and turn around the steering axis passing through the ground contact point. (c) Omni wheel, which can move in a direction perpendicular to the wheel plane by using free rollers along the circumference. (d) Mecanum wheel, which has free rollers with axes tilted from the wheel plane. (e) Caster wheel, which has an offset between the axle of the wheel and the steering axis.

motors are installed. Thus, the proposed wheel mechanism establishes both the superb mobility of the omnidirectional mobile robot and the reliability of the conventional mobile robot at the same time.

In this paper, we first introduce the novel principle for omnidirectional movement with a passive sliding joint through the discussion of the kinematic conditions. Then we propose a novel omnidirectional mobile robot by using the principle. Next, a kinematic analysis, a reachable region evaluation, simulation of unlimited translation, and demonstrative experiments are presented, and the effectiveness of the proposed omnidirectional mobile robot is verified.

\section{Novel OmNidirectional Mobile Robot}

In this section, we propose the novel wheel mechanism for omnidirectional movement and the novel omnidirectional mobile robot using the mechanism.

\section{A. Synthesis of Novel Wheel Mechanism: Slidable Wheel}

This section shows the embodiment process of a novel principle for omnidirectional movement into a wheel mechanism based on the analysis of the kinematic conditions. In order to discuss the kinematics common to all types of wheels, including the fixed wheel, orientable wheel, omni wheel, Mecanum wheel, and caster wheel, we consider the motion of a mobile robot with each wheel type. As shown in Fig. 2(a), the global reference frame $O-X_{I} Y_{I}$ is fixed on the floor. The pose of the local reference frame $P-X_{R} Y_{R}$ in the global frame is represented by a vector $\xi$, as follows.

$\xi=\left[\begin{array}{l}x \\ y \\ \theta\end{array}\right]$

In the local reference frame, as shown in Fig. 2(b), the wheel of the mobile robot is located at a distance $l$ from the origin $P$ and at an angle $\alpha$ from $X_{R}$. The axis of the wheel is tilted at an angle $\beta$ from a line passing through $P$ and the center of the wheel.

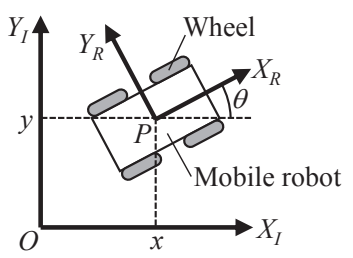

(a)

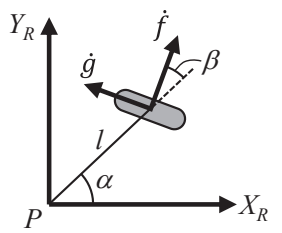

(b)
Fig. 2. Reference frames for kinematic analysis of a mobile robot. (a) Position and orientation of the mobile robot in the global reference frame. (b) Position and orientation of the wheel in the local reference frame.

Parameters $\dot{g}$ and $\dot{f}$ denote the velocity of the wheel in the primary direction and the direction perpendicular to it, respectively. Here, the primary direction means the movement direction by rotation of the wheel around the axle, namely, the left and right directions in Fig. 1. Then, the kinematic equations for the primary and perpendicular directions are represented as follows:

$[\mathrm{s}(\alpha+\beta)-\mathrm{c}(\alpha+\beta)-l \mathrm{c} \beta] R(\theta) \dot{\xi}+\dot{g}=0$

$\left[\begin{array}{lll}\mathrm{c}(\alpha+\beta) & \mathrm{s}(\alpha+\beta) & l \mathrm{~s} \beta\end{array}\right] R(\theta) \dot{\xi}-\dot{f}=0$

where the rotation matrix $R(\theta)$ transforms the vector in the global reference frame to the vector in the local frame, $\mathrm{c}$ is the cosine, and $\mathrm{s}$ is the sine.

$R(\theta)=\left[\begin{array}{ccc}\mathrm{c} \theta & \mathrm{s} \theta & 0 \\ -\mathrm{s} \theta & \mathrm{c} \theta & 0 \\ 0 & 0 & 1\end{array}\right]$

When the wheel is a fixed wheel [Fig. 1(a)] with radius $r$ and rotation angle $\varphi$, its velocity is $\dot{g}=r \dot{\varphi}$ in (2). Because the rotational velocity $\dot{\varphi}$ is arbitrary, (2) is satisfied at any velocity $\dot{\xi}$. On the other hand, the constraint that the wheel does not skid leads to $\dot{f}=0$ in (3). Then, (3) becomes a nonholonomic constraint, i.e., the components of $\dot{\xi}$ are subordinate to each other, and the mobile robot is no longer able to move at an arbitrary velocity with three-degree-of-freedom (three-DOF) planar motion. Therefore, robots with fixed wheels are nonholonomic, and not omnidirectional.

In contrast, if both $\dot{g}$ and $\dot{f}$ can take arbitrary values, neither (2) nor (3) yields nonholonomic constraints. The equations are established at any $\dot{\xi}$ by substituting appropriate values in $\dot{g}$ and $\dot{f}$. A mobile robot whose wheels satisfy these conditions and provide three or more active DOFs can be an omnidirectional mobile robot. For example, the kinematic equations of the omni wheel [Fig. 1(c)] are given by substituting $\dot{g}=r \dot{\varphi}$ and $\dot{f}=$ $-r_{r} \dot{\varphi}_{r}$ [30], [31]. Here, $r_{r}$ and $\varphi_{r}$ are the radius and rotation angle of the free rollers, respectively. The value of $\dot{\varphi}_{r}$, as well as $\dot{\varphi}$, is arbitrary because the free rollers of the omni wheel can rotate freely. Therefore, a robot with three or more omni wheels can be an omnidirectional mobile robot. The Mecanum wheel [Fig. 1(d)] is almost the same as the omni wheel. The caster wheel [Fig. 1(e)] has an offset distance $d$ between the ground contact point and the steering axis, so that $\dot{g}=r \dot{\varphi}$ and $\dot{f}=$ $d(\dot{\beta}-\dot{\theta})$ are given [30], [31]. Because $\dot{\theta}$ in $\dot{f}$ is a component of $\dot{\xi}, \dot{\xi}$ is arbitrary if the steering angular velocity $\dot{\beta}$ is arbitrary. 


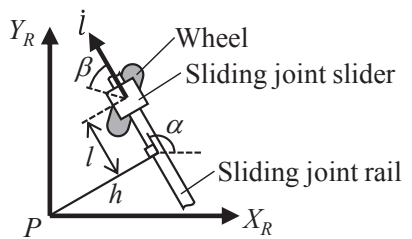

Fig. 3. Proposed wheel mechanism using a sliding joint, slidable wheel, and its parameters.

Therefore, a mobile robot with using caster wheels can also be an omnidirectional mobile robot.

As shown above, $\dot{g}$ generally includes $\dot{\varphi}$ and then $\dot{g}$ becomes arbitrary whenever the wheel can rotate around its axle at an arbitrary velocity. Therefore, we consider the mechanism to allow $\dot{f}$ to take an arbitrary value and synthesize a novel wheel mechanism for omnidirectional movement by adopting other parameters as $\dot{f}$ rather than $\dot{\varphi}_{r}$ or $\dot{\beta}$ in the existing mechanisms. When we assume the use of the fixed or orientable wheel in order to keep the structure simple and reliable, either $\alpha$ or $l$ can be used in Fig. 2(b); if $\alpha$ is chosen as a variable, however, the effect of $\dot{\alpha}$ in $\dot{f}$ is close to that of $\dot{\beta}$ in the caster wheel. For that reason, we focus on the wheel mechanism in which $l$ is a variable and $l$ is used for $\dot{f}$.

Here, we propose a slidable wheel, whose sliding joint enables $l$ to vary. Fig. 3 shows the schematic structure and parameters for the slidable wheel. The rail of the sliding joint is located at a distance $h$ from $P$ and tilted at an angle $\alpha$ from $X_{R}$. The conventional wheel can move passively along the sliding joint relative to the rail. The wheel is at distance $l$ on the sliding joint from the foot of the perpendicular from $P$. The angle between the wheel axle and the sliding joint rail is $\beta$, whose value is either fixed or changed actively. Then, the kinematic equations equivalent to (2) and (3) are as follows:

$\left[\begin{array}{lll}\mathrm{s}(\alpha+\beta)-\mathrm{c}(\alpha+\beta) & h \mathrm{~s} \beta-l \mathrm{c} \beta\end{array}\right] R(\theta) \dot{\xi}+r \dot{\varphi}+\dot{l} \mathrm{~s} \beta=0$

$[\mathrm{c}(\alpha+\beta) \mathrm{s}(\alpha+\beta) \quad h \mathrm{c} \beta+l \mathrm{~s} \beta] R(\theta) \dot{\xi}+\dot{l} \mathrm{c} \beta=0$.

In (5) and (6), $\dot{g}=r \dot{\varphi}+(\dot{l}+h \dot{\theta}) \mathrm{s} \beta$ and $\dot{f}=-(i+h \dot{\theta}) \mathrm{c} \beta$ are given. By considering $\dot{l}$ as arbitrary, (5) and (6) generate zero nonholonomic constraints for $\dot{\xi}$ under the condition $c \beta \neq$ 0 . Thus, the kinematic conditions are applicable to any $\dot{\xi}$, and so omnidirectional mobile robots can be realized by using three or more slidable wheels.

\section{B. Structure and Motion of Slidable-Wheeled Omnidirectional Mobile Robot}

We propose a novel omnidirectional mobile robot with three slidable wheels. This robot, named SWOM (slidable-wheeled omnidirectional mobile robot), is shown in Fig. 4. The main body of SWOM consists of a base block and three rails of sliding joints. The three rails are fixed to the base block at 120-degree intervals. Each driving unit of SWOM is like an orientable wheel with a slider of the sliding joint. The wheel rotates actively around both the axle and the ground contact

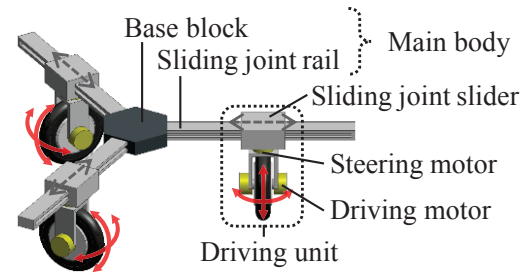

Fig. 4. Proposed omnidirectional mobile robot, SWOM.

point by the driving motor and the steering motor, respectively. Through the passive sliding joints, the driving units can move relative to the rail or the main body.

We next consider the omnidirectional movement of SWOM. Fig. 5 schematically shows the motion of SWOM: (a) moving forward, (b) moving to the right, (c) moving diagonally forward right, and (d) turning. Note that the upward direction in the figure is the forward direction. Each driving unit can move in the primary direction and steer the wheel, but cannot move in the perpendicular direction. However, the relative movements of the sliding joints enable the main body of SWOM to move in the direction perpendicular to each wheel plane. As a result, the main body is capable of three-DOF planar motion. In addition, the steering angle of each wheel is constant. This means that the motion in Fig. 5 can be switched from one to another immediately, without steering the wheels. Thus, SWOM achieves omnidirectional movement.

In Fig. 5, the movement direction of each wheel is perpendicular to the sliding joint. In other cases, SWOM can make omnidirectional movement. For example, Fig. 6 shows the motion of SWOM when all wheels are in the forward and backward direction.

As shown in Figs. 5 and 6, the omnidirectional movement of SWOM depends on the relative movements of the sliding joints. Therefore, the reachable region is limited to the region where the relative movements are feasible, i.e., where the sliders of the sliding joints are away from the ends of the rails. Here, we consider another movement method, shown in Fig. 7, in which the steering angles of the wheels are changed according to the movement direction of the main body. The movement direction of each wheel is the same as that of the main body during the translation, such as in (a) moving forward, (b) moving to the right, and (c) moving diagonally forward right, or the circumference direction of the rotation in (d) turning. In this way, SWOM can move in each direction without relative movements of the sliding joints. Therefore, the movable range of the sliding joints does not restrict the reachable region of SWOM, and so SWOM can continue with movement unlimitedly. On the other hand, when changing the movement direction, e.g., when switching the motion from Fig. 7(a) to (b), SWOM has to steer the wheels. This means that SWOM performs as a nonholonomic mobile robot with three orientable wheels, and is unable to make omnidirectional movement. The state in Fig. 7 is different from that in Figs. 5 or 6.

As suggested above, the method shown in Figs. 5 and 6 enables omnidirectional movement inside the limited reachable region. In contrast, the method shown in Fig. 7 allows SWOM to move unlimitedly in each direction with nonholonomic constraints. To realize unlimited and omnidirectional 


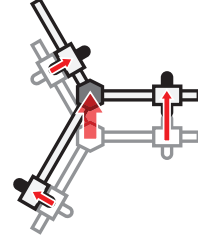

(a) (b)

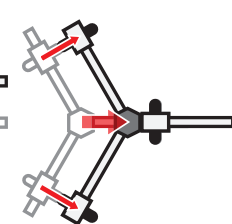

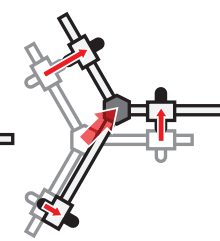

(c)

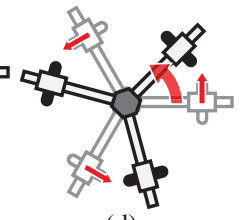

(d)
Fig. 5. Omnidirectional movement of SWOM when the movement direction of each wheel is perpendicular to the sliding joint. (a) Moving forward. (b) Moving to the right. (c) Moving diagonally forward right. (d) Turning. The upward direction in the figure is the forward direction.

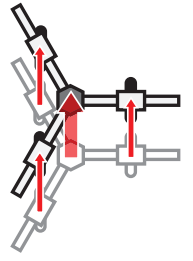

(a)

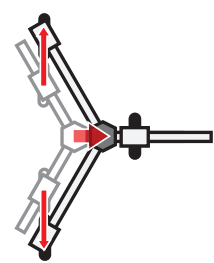

(b)

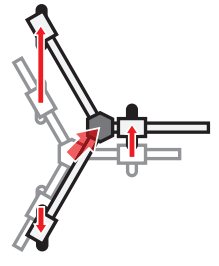

(c)

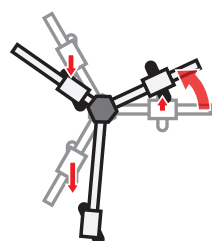

(d)
Fig. 6. Omnidirectional movement of SWOM when the movement direction of each wheel is parallel to the forward and backward direction. (a) Moving forward. (b) Moving to the right. (c) Moving diagonally forward right. (d) Turning. The upward direction in the figure is the forward direction.

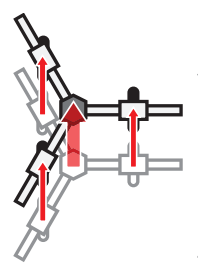

(a)

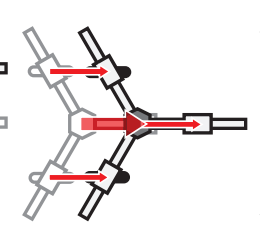

(b)

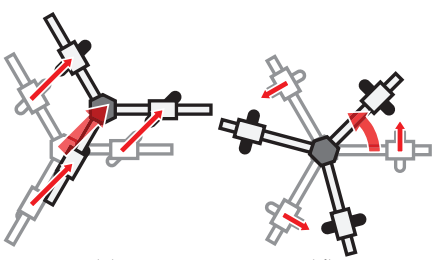

(c) (d)
Fig. 7. Movement of SWOM when the movement direction of each wheel is changed depending on the movement direction of the main body. (a) Moving forward. (b) Moving to the right. (c) Moving diagonally forward right. (d) Turning. The upward direction in the figure is the forward direction.

movement, it is reasonable to combine these two movement methods. Specifically, SWOM starts moving by using the former method and shifts to the latter method by steering the wheels while moving before the driving units reach the ends of the rails. Thus, SWOM can immediately move an arbitrary distance in an arbitrary direction from an arbitrary initial condition.

\section{Differences between SWOM and Other Omnidirectional Mobile Robots}

First, we compare SWOM with an omni-wheeled omnidirectional mobile robot. (i) The omni wheel has the problem of a low load capacity due to its complicated structure, unavoidable vertical vibration due to the discontinuous ground contact point, and low surmountable bumps due to the small radius of the free rollers. In contrast, SWOM uses conventional wheels that are free from these problems. (ii) Omni wheels have difficulties also in applying dead reckoning or feedback control to mobile robots because it is difficult to measure the passive rotation of the free rollers. However, it is easy to install sensors in SWOM to measure the rotation and steering of the wheels or the relative movements of the sliding joints.

Next, we contrast SWOM and a mobile robot with caster wheels. (iii) Both use conventional wheels, but the steering axis of the caster wheel is off-center from the ground contact point, whereas the steering axis of the slidable wheel passes through the ground contact point. Thus, the caster wheel becomes unstable when the movement direction is reversed; the wheel may be rotated around the steering axis so quickly that it makes motion errors. On the other hand, the driving unit of SWOM can avoid this problem because it has a plane-symmetrical shape in the primary direction. (iv) When both the wheel axles and steering axes are motorized, an omnidirectional mobile robot with two or more caster wheels causes redundancy, and so the motors must be driven with high accuracy. However, SWOM can steer the wheels independently from the motion of the main body, and even the three slidable wheels never cause such an overconstraint-like situation.

The above comparisons show that the main difference between SWOM and other omnidirectional mobile robots is the wheel mechanism. SWOM utilizes slidable wheels, whereas others utilize omni wheels or caster wheels. This difference makes the structure of SWOM simple and provides benefits in increasing the reliability of movement as explained in (i)-(iv). Then, SWOM can solve the problems of the omnidirectional mobile robots that have been proposed in the past.

\section{KINEMATIC ANALYSIS OF SWOM}

\section{A. Kinematics}

Fig. 8 shows the coordinate systems for the kinematic analysis of SWOM. The reference frames are basically the same as those in Fig. 2, except the origin of the local reference frame $P$ is on the center of the base block, and $X_{R}$ is parallel to one of the sliding joints. We assign the driving unit in the sliding joint parallel to $X_{R}$ as driving unit 1, and the others as driving units 2 and 3 counterclockwise.

First, we explain the kinematics by pairing a sliding joint and a driving unit. By substituting $h=0$ in (5) and (6), we obtain the following six conditional expressions, where subscript $i$ represents the parameter belonging to driving unit $i$.

$$
\begin{aligned}
& {\left[\mathrm{s}\left(\alpha_{i}+\beta_{i}\right)-\mathrm{c}\left(\alpha_{i}+\beta_{i}\right)-l_{i} \mathrm{c} \beta_{i}\right] R(\theta) \dot{\xi}+r_{i} \dot{\varphi}_{\imath}+\dot{l}_{\imath} \mathrm{s} \beta_{i}=0} \\
& {\left[\begin{array}{lll}
\mathrm{c}\left(\alpha_{i}+\beta_{i}\right) & \mathrm{s}\left(\alpha_{i}+\beta_{i}\right) & l_{i} \mathrm{~s} \beta_{i}
\end{array}\right] R(\theta) \dot{\xi}+\dot{l}_{l} \mathrm{c} \beta_{i}=0}
\end{aligned}
$$

Here, $\alpha_{1}=0, \alpha_{2}=2 \pi / 3$, and $\alpha_{3}=4 \pi / 3$ are constants. If the rotational velocity of the wheel $\dot{\varphi}_{i}$ and the relative velocity of the driving unit on the sliding joint $\dot{l}_{i}$ are arbitrary, (7) and (8) give zero nonholonomic constraints for $\dot{\xi}$ under the condition $\beta_{i} \neq \pm \pi / 2$. This proves theoretically that SWOM can move at an arbitrary velocity with regard to three-DOF planar motion and can perform as an omnidirectional mobile robot.

By eliminating $\dot{l}_{i}$ from (7) and (8), the following equation is obtained.

$\left[\begin{array}{ccc}-\mathrm{s} \alpha_{1} & \mathrm{c} \alpha_{1} & l_{1} \\ -\mathrm{s} \alpha_{2} & \mathrm{c} \alpha_{2} & l_{2} \\ -\mathrm{s} \alpha_{3} & \mathrm{c} \alpha_{3} & l_{3}\end{array}\right] R(\theta) \dot{\xi}-\left[\begin{array}{ccc}\mathrm{c} \beta_{1} & 0 & 0 \\ 0 & \mathrm{c} \beta_{2} & 0 \\ 0 & 0 & \mathrm{c} \beta_{3}\end{array}\right]\left[\begin{array}{l}r_{1} \dot{\varphi}_{1} \\ r_{2} \dot{\varphi}_{2} \\ r_{3} \dot{\varphi}_{3}\end{array}\right]=0$ 
Fig. 8. SWOM and reference frames.

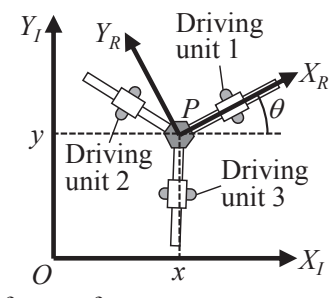

The forward and inverse kinematics are represented by (10) and (11), respectively, when the input is the velocities of the driving units and the output is the velocity of the main body. Here, $\beta_{i}=$ $\pm \pi / 2$ is excluded in (11).

$\dot{\xi}=R(\theta)^{-1}\left[\begin{array}{lll}-\mathrm{s} \alpha_{1} & \mathrm{c} \alpha_{1} & l_{1} \\ -\mathrm{s} \alpha_{2} & \mathrm{c} \alpha_{2} & l_{2} \\ -\mathrm{s} \alpha_{3} & \mathrm{c} \alpha_{3} & l_{3}\end{array}\right]^{-1}\left[\begin{array}{ccc}\mathrm{c} \beta_{1} & 0 & 0 \\ 0 & \mathrm{c} \beta_{2} & 0 \\ 0 & 0 & \mathrm{c} \beta_{3}\end{array}\right]\left[\begin{array}{l}r_{1} \dot{\varphi}_{1} \\ r_{2} \dot{\varphi}_{2} \\ r_{3} \dot{\varphi}_{3}\end{array}\right]$

$\left[\begin{array}{c}r_{1} \dot{\varphi}_{1} \\ r_{2} \dot{\varphi}_{2} \\ r_{3} \dot{\varphi}_{3}\end{array}\right]=\left[\begin{array}{ccc}\mathrm{c} \beta_{1} & 0 & 0 \\ 0 & \mathrm{c} \beta_{2} & 0 \\ 0 & 0 & \mathrm{c} \beta_{3}\end{array}\right]^{-1}\left[\begin{array}{lll}-\mathrm{s} \alpha_{1} & \mathrm{c} \alpha_{1} & l_{1} \\ -\mathrm{s} \alpha_{2} & \mathrm{c} \alpha_{2} & l_{2} \\ -\mathrm{s} \alpha_{3} & \mathrm{c} \alpha_{3} & l_{3}\end{array}\right] R(\theta) \dot{\xi}$

\section{B. Reachable Region under Fixed Steering Angles}

The reachable region of SWOM is limited by the movable range of the sliding joints when SWOM makes omnidirectional movement with fixed steering angles. In this section, we evaluate the reachable region quantitatively. The analysis target excludes the singular configuration, $\beta_{i}= \pm \pi / 2$.

First, we examine the translation without rotation of SWOM. If $\theta=\dot{\theta}=0$ is given in (8) and the global and local reference frames are in agreement at the initial condition, the relative position of the driving unit on the sliding joint $l_{i}$ is given as follows:

$l_{i}=-\frac{\mathrm{c}\left(\alpha_{i}+\beta_{i}\right)}{\mathrm{c} \beta_{i}} x-\frac{\mathrm{s}\left(\alpha_{i}+\beta_{i}\right)}{\mathrm{c} \beta_{i}} y+l_{i 0}$

where $l_{i 0}$ denotes the relative position of driving unit $i$ on the sliding joint at the initial condition. When the movable range of the sliding joints is represented by $l_{\min } \leq l_{i} \leq l_{\max }$, the reachable region of the main body of SWOM is obtained.

$-\left(l_{i 0}-l_{\min }\right) \leq-\frac{\mathrm{c}\left(\alpha_{i}+\beta_{i}\right)}{\mathrm{c} \beta_{i}} x-\frac{\mathrm{s}\left(\alpha_{i}+\beta_{i}\right)}{\mathrm{c} \beta_{i}} y \leq l_{\max }-l_{i 0}$

Under the conditions $\beta_{1}=\beta_{2}=\beta_{3}=0$ and $l_{10}=l_{20}=l_{30}$, the reachable region of the main body in translation, represented by the center of the base block, is shown in Fig. 9(a). The six inequalities of (13) shape the reachable region into a hexagon. The movable range of the sliding joint connected with driving unit $i$ defines two boundaries of the region. The boundaries are parallel to the wheel plane of driving unit $i$ and apart from the center of the base block by $l_{\max }-l_{i 0}$ and $l_{i 0}-$ $l_{\min }$. When $\beta_{i}$ and $l_{i 0}$ are varied, as shown in Fig. 9(b), a reachable region remains in all directions, while the reachable distance depends on the direction. In the case that the movement directions of the three wheels match each other, as

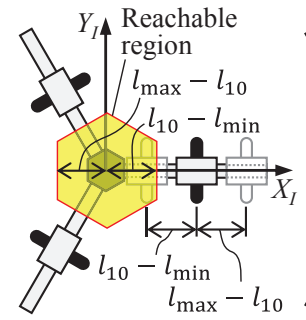

(a)

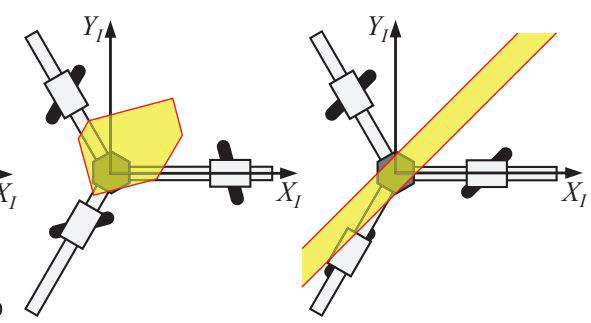

(b)

(c)
Fig. 9. Reachable region in translation of SWOM with fixed steering angles of the wheels. (a) $\beta_{1}=\beta_{2}=\beta_{3}=0, l_{10}=l_{20}=l_{30}$. (b) $\beta_{1}=\pi / 12, \beta_{2}=$ $\pi / 6, \beta_{3}=\pi / 4, l_{10}>l_{20}>l_{30}$. (c) $\alpha_{1}+\beta_{1}=\alpha_{2}+\beta_{2}=\alpha_{3}+\beta_{3}=\pi / 4$, $l_{10}=l_{20}=l_{30}$.

shown in Fig. 9(c), the gradients of the six boundaries of (13) are equivalent. As a result, the reachable region becomes open, and SWOM can move unlimitedly in the movement direction of the wheel. In the other directions, the reachable distance is limited. Thus, in any direction of the wheels, except for the singular configuration, a reachable region exists in all directions and SWOM can be translated in any direction immediately.

Next, we consider the reachable region when SWOM rotates around the center of the base block without translation. When $x=\dot{x}=y=\dot{y}=0$ is given in (8) and the initial condition is the same as in the translation discussed above, $l_{i}$ is obtained:

$l_{i}=l_{i 0} \exp \left[-\mathrm{t} \beta_{i} \theta\right]$

where $t$ is the tangent. Therefore, the reachable region of the main body of SWOM is represented by the following inequalities.

$$
\left\{\begin{array}{rlrl}
-\frac{1}{\mathrm{t} \beta_{i}} \ln \frac{l_{\min }}{l_{i 0}} & \leq \theta \leq-\frac{1}{\mathrm{t} \beta_{i}} \ln \frac{l_{\max }}{l_{i 0}}, & & -\frac{\pi}{2}<\beta_{i}<0 \\
-\infty & <\theta<\infty, & \beta_{i}=0 \\
-\frac{1}{\mathrm{t} \beta_{i}} \ln \frac{l_{\max }}{l_{i 0}} \leq \theta \leq-\frac{1}{\mathrm{t} \beta_{i}} \ln \frac{l_{\min }}{l_{i 0}}, & & 0<\beta_{i}<\frac{\pi}{2}
\end{array}\right.
$$

Equation (15) shows SWOM can rotate in either direction with any steering angle of the wheels except for the singular configuration.

\section{Singular Configuration}

SWOM falls into the singular configuration when the movement direction of the wheel is parallel to the rail of the sliding joint. This is because the motion of the driving unit is never transmitted to the main body through the sliding joint. When driving unit $i$ causes the singular configuration, $\beta_{i}=$ $\pm \pi / 2$, and the velocity of the main body is replaced by $R(\theta) \dot{\xi}=\left[\begin{array}{lll}\dot{X} & \dot{Y} & \dot{\theta}\end{array}\right]^{\mathrm{T}},(8)$ gives a nonholonomic constraint.

$\mathrm{s} \alpha_{i} \dot{X}-\mathrm{c} \alpha_{i} \dot{Y}-l_{i} \dot{\theta}=0$

Every driving unit with $\beta_{i}= \pm \pi / 2$ generates this constraint, and reduces the DOFs of the main body by one DOF, so that omnidirectional movement is no longer feasible. For example, when driving unit 1 is in the singular configuration, the DOFs 
of SWOM is two: translation in the $X_{R}$ direction and rotation around driving unit 1 . When two driving units fall into the singular configuration at the same time, SWOM motion is only one-DOF rotation around a certain point. Thus, the movable direction is restricted in the singular configuration, but SWOM can move in the accepted directions. When all the driving units are in the singular configuration, SWOM cannot move at all. However, SWOM can avoid the singular configuration just by steering the driving units slightly from $\beta_{i}= \pm \pi / 2$.

\section{Simulation of Unlimited Translation}

In this section, the unlimited omnidirectional movement process explained in Section II-B is discussed. In order to practically demonstrate the process, unlimited translation of SWOM is simulated.

\section{A. Fundamental Method of Unlimited Omnidirectional Movement}

First, we explain the basic idea for the unlimited omnidirectional movement of SWOM by considering the transition from a certain initial condition to a target condition where SWOM moves at a certain desired velocity. In the initial condition, the steering angles, the rotational velocities, and the steering velocities of the wheels are arbitrary. First, we accelerate the main body to the target velocity by adjusting the rotational velocities and the steering velocities of the wheels according to (11). As shown in Section III-B, the reachable region where SWOM can move immediately without steering the wheels exists in all directions, regardless of the directions of the wheels (except in the singular configuration). Thus, SWOM can accelerate to the target velocity immediately inside the reachable region. At the same time that SWOM moves, we change the steering angles of the wheels. The reachable region changes depending on the steering angles, as shown in Fig. 9, but SWOM can continue moving by keeping at least the target direction in the reachable region at each instant. At the end of the transition, the steering angles of the wheels are fixed at the angles that enable SWOM to move unlimitedly, as in Fig. 7. Then, the target condition is achieved. In this way, SWOM can start moving immediately with arbitrary steering angles, rotational velocities, and steering velocities of the wheels, and continue moving unlimitedly in an arbitrary direction.

In the subsequent sections, we deal with unlimited translation. We define the state of unlimited translation as the state where the directions of all wheels are parallel and SWOM can move in a direction unlimitedly, as in Fig. 7(a)-(c). We assume that the initial condition is the state of unlimited translation in a certain initial direction and the target condition is the state of unlimited translation in another target direction. We explain the method for generating the trajectory of the driving units to keep SWOM in the reachable region during the transition of the movement direction in unlimited translation.

\section{B. Trajectories Satisfying Constraints}

To perform unlimited translation, we examine the SWOM trajectory that satisfies the constraints. By solving (8), $\beta_{i}$ is obtained.

$\beta_{i}=\tan ^{-1}\left(\frac{\mathrm{c} \alpha_{i} \dot{X}+s \alpha_{i} \dot{Y}+\dot{l}_{l}}{s \alpha_{i} \dot{X}-c \alpha_{i} \dot{Y}-l_{i} \dot{\theta}}\right)$

According to (17), $\beta_{i}$ becomes continuous when $\dot{X}, \dot{Y}, \dot{\theta}$, and $i_{l}$ are continuous, because the argument of the arctangent on the right side becomes continuous, except for $\mathrm{s} \alpha_{i} \dot{X}-\mathrm{c} \alpha_{i} \dot{Y}-$ $l_{i} \dot{\theta}=0$. By using (9), the discontinuous condition can be rewritten as follows:

$r_{i} \dot{\varphi}_{l} \mathrm{c} \beta_{i}=0$.

Thus, SWOM can move continuously when $\beta_{i}$ is in the trajectory where $\dot{X}, \dot{Y}, \dot{\theta}$, and $\dot{l}_{l}$ are continuous and neither $\dot{\varphi}_{l}=0$ nor $\beta_{i}= \pm \pi / 2$ is passed through. Additionally, the steering angle is arbitrary when the wheel is stopping, so that $\dot{\varphi}_{l}=0$ can be passed through when $\beta_{i}$ is continuous before and after $\dot{\varphi}_{l}=0$. We deal with the singular configuration $\beta_{i}=$ $\pm \pi / 2$ in Section IV-D, and assume $\beta_{i} \neq \pm \pi / 2$ in this section.

If we consider (8) as a differential equation with respect to $l_{i}$, the integration form is as follows.

$$
\begin{array}{r}
l_{i}=\left[\int\left\{-\frac{\mathrm{c}\left(\alpha_{i}+\beta_{i}\right)}{\mathrm{c} \beta_{i}} \dot{X}-\frac{\mathrm{s}\left(\alpha_{i}+\beta_{i}\right)}{\mathrm{c} \beta_{i}} \dot{Y}\right\} \exp \left(\int \mathrm{t} \beta_{i} \dot{\theta} d t\right) d t+C\right] \\
\cdot \exp \left(-\int \mathrm{t} \beta_{i} \dot{\theta} d t\right)
\end{array}
$$

where $C$ denotes the integral constant. It is impossible to calculate the integration of (19) analytically, because the slidable wheels of SWOM are nonholonomic. As shown in (12) and (14), however, we can obtain the general solution in the case that SWOM trajectories are either translation or rotation only.

By using the equations derived above, the trajectories of the driving units are obtained through the following process. First, we calculate the target value of $\beta_{i}$ in the target condition by using (17). Next, we find the trajectory of $\beta_{i}$ that satisfies the boundary conditions such that $\beta_{i}, \dot{\beta}_{l}$, and $l_{i}$ are continuous by using (19). Finally, $\dot{\varphi}_{l}$ is given by substituting the calculated $\beta_{i}$ and $l_{i}$ in (11). Thus, the trajectories of rotation and steering of the wheels between the initial and target conditions of SWOM are provided. The trajectories should be chosen to satisfy the condition that SWOM is inside the reachable region, i.e., $l_{\min }<l_{i}<l_{\max }$ and $\beta_{i} \neq \pm \pi / 2$.

\section{Change of Movement Direction in Unlimited Translation}

We now show an example of the trajectory generation of unlimited translation by using the expressions obtained in the previous section. We divide the process of transition from the initial condition to the target condition into two steps. Additionally to $\theta=\dot{\theta}=0$, we give a part of the boundary conditions and the trajectories as follows. 
$\dot{x}(t)= \begin{cases}V_{0} \mathrm{c} \chi_{0}, & t=t_{0} \\ V_{2} \mathrm{c} \chi_{2}, & t_{1} \leq t \leq t_{2}\end{cases}$
$\dot{y}(t)= \begin{cases}V_{0} \mathrm{~s} \chi_{0}, & t=t_{0} \\ V_{2} \mathrm{~s} \chi_{2}, & t_{1} \leq t \leq t_{2}\end{cases}$
$\beta_{i}(t)= \begin{cases}\beta_{i 0}, & t_{0} \leq t \leq t_{1} \\ \beta_{i 2}, & t=t_{2}\end{cases}$
$l_{i}\left(t_{0}\right)=l_{i 0} l_{i}\left(t_{2}\right)=l_{i 2}$
$\dot{\beta}_{l}\left(t_{1}\right)=\dot{\beta}_{l}\left(t_{2}\right)=i_{l}\left(t_{0}\right)=i_{l}\left(t_{2}\right)=0$

SWOM takes the initial condition, i.e., the state of unlimited translation in the initial direction for $t \leq t_{0}$, and then switches from moving in the initial direction to changing the direction to the target direction at $t=t_{0}$. First, for $t_{0} \leq t \leq t_{1}$, the main body of SWOM accelerates to the target velocity inside the reachable region with the directions of the wheels fixed. Next, for $t_{1} \leq t \leq t_{2}$, the driving units change $\beta_{i}$ and control $i_{l}$ while keeping the velocity of the main body. Finally, SWOM achieves the target condition at $t=t_{2}$ and then continues unlimited translation in the target direction. Here, $V_{0}$ and $V_{2}$ are the velocities of the main body at $t=t_{0}$ and $t=t_{2}$, respectively; $\chi_{0}$ and $\chi_{2}$ denote the angles between $X_{I}$ and the initial or target direction, respectively.

The steering angles of the wheels in the state of unlimited translation, $\beta_{i 0}$ and $\beta_{i 2}$, are obtained from (17).

$\beta_{i 0}=\chi_{0}-\alpha_{i} \pm \frac{\pi}{2} \quad \beta_{i 2}=\chi_{2}-\alpha_{i} \pm \frac{\pi}{2}$

The plus/minus signs in (20) correspond to the reverse and forward rotation of the wheels, but are not distinguished structurally in SWOM. Then, we choose $\beta_{i 0}$ or $\beta_{i 2}$ that satisfies $-\pi / 2<\beta_{i}(t)<\pi / 2$. Next, $l_{i}(t)$ is calculated from (19) by considering the boundary conditions.

$$
\begin{aligned}
& l_{i}= \\
& \left\{\begin{array}{r}
-\frac{\mathrm{c}\left(\alpha_{i}+\beta_{i 0}\right)}{\mathrm{c} \beta_{i 0}}\left(x-x_{0}\right)-\frac{\mathrm{s}\left(\alpha_{i}+\beta_{i 0}\right)}{\mathrm{c} \beta_{i 0}}\left(y-y_{0}\right)+l_{i 0}, \quad t_{0} \leq t \leq t_{1} \\
-V_{2} \mathrm{c}\left(\alpha_{i}-\chi_{2}\right)\left(t-t_{1}\right)+V_{2} \mathrm{~s}\left(\alpha_{i}-\chi_{2}\right) \int_{t_{1}}^{t} \mathrm{t} \beta_{i}(\tau) d \tau+l_{i 1}, \\
t_{1}<t \leq t_{2}
\end{array}\right.
\end{aligned}
$$

where $x\left(t_{0}\right)=x_{0}, y\left(t_{0}\right)=y_{0}$, and $l_{i}\left(t_{1}\right)=l_{i 1}$ are constants. As shown in (21), the analytic solutions for this trajectory are given when $\mathrm{t} \beta_{i}(t)$ can be integrated, i.e., when $\beta_{i}(t)$ satisfies $-\pi / 2<\beta_{i}(t)<\pi / 2$ for $t_{1} \leq t \leq t_{2}$. By considering the continuities of $\beta_{i}(t)$ and $l_{i}(t)$ at $t=t_{1}, t_{2}$, the boundary conditions $\beta_{i}(t)$ should satisfy the following five expressions.

$$
\begin{gathered}
\beta_{i}\left(t_{1}\right)=\beta_{i 0} \quad \beta_{i}\left(t_{2}\right)=\beta_{i 2} \quad \dot{\beta}_{l}\left(t_{1}\right)=\dot{\beta}_{l}\left(t_{2}\right)=0 \\
\int_{t_{1}}^{t_{2}} \mathrm{t} \beta_{i}(t) d t=\frac{\left(l_{i 2}-l_{i 1}\right)+V_{2} \mathrm{c}\left(\alpha_{i}-\chi_{2}\right)\left(t_{2}-t_{1}\right)}{V_{2} \mathrm{~s}\left(\alpha_{i}-\chi_{2}\right)}
\end{gathered}
$$

In the following simulation, we suppose $t \beta_{i}(t)$ is a quartic function satisfying (22). Thus, $\beta_{i}(t)$ and $l_{i}(t)$ are determined, and then the rotational velocity of the wheels $\dot{\varphi}_{l}(t)$ is obtained from (11).

We next show the results of the numerical simulation. We set the values of the parameters as follows: $t_{0}=0.0 \mathrm{~s}, t_{1}=2.0 \mathrm{~s}$, $t_{2}=7.0 \mathrm{~s} ; \quad V_{0}=0 \mathrm{~mm} / \mathrm{s}, \quad V_{2}=20 \mathrm{~mm} / \mathrm{s} ; \quad l_{i 0}=l_{i 2}=$ $300 \mathrm{~mm}, \quad l_{\min }=100 \mathrm{~mm}, \quad l_{\max }=500 \mathrm{~mm} ; \quad x_{0}=y_{0}=$ $0 \mathrm{~mm}$; and $\chi_{0}=-\pi / 6, \chi_{2}=\pi / 2$. The movement velocities $\dot{x}(t)$ and $\dot{y}(t)$ for $t_{0} \leq t \leq t_{1}$ are linear functions that are continuous at $t=t_{0}, t_{1}$. These conditions give the trajectories shown in Fig. 10. Fig. 10(a) shows the trajectories of the main body and three driving units. Fig. 10(b) shows the change of $l_{i}$ and $\beta_{i}$ over time.

For $t=0.0-2.0 \mathrm{~s}$, by accelerating the wheels while maintaining the initial steering angles, the main body of SWOM starts to move in the target direction with the relative motion of the sliding joints inside the reachable region. For $t=$ $2.0-7.0 \mathrm{~s}$, the directions of the wheels are changing. The main body moves at a constant velocity in the target direction with the relative positions of the driving units controlled. After $t=$ $7.0 \mathrm{~s}$, the directions of the wheels and the relative positions of the driving units are all constant; this is the state of unlimited translation in the target direction. As a result, although the trajectories of the driving units are smooth curves, the main body moves straight from the beginning of the change of the movement direction. That is, the main body moves in the target direction immediately in the initial condition. In this simulation, we also set the boundary conditions so that the relative positions of the driving units at the initial and target conditions are in agreement. By keeping the driving units as far from the ends of the sliding joints as possible, the driving units are likely to stay within the movable range of the sliding joints in the following movement.

The above results verify that the appropriate trajectory continuously connects the state of unlimited translation in a certain direction with that in another direction.

\section{Singular Configuration in Unlimited Translation}

Finally, we discuss handling of the singular configuration. When the initial and target conditions are not singular in unlimited translation, SWOM can definitely choose trajectories between them that do not pass through $\beta_{i}= \pm \pi / 2$, and so can avoid the singular configuration. In the following, we consider the case that the initial or target condition is in the singular configuration.

If the target condition is singular, the same strategy as the nonsingular case is available immediately before the target condition, when the movement direction of the main body becomes parallel to the sliding joint connected with the driving unit that is singular in the target condition. At this point, the relative movement of the sliding joint allows the driving unit to stop temporarily without interruption of movement of the main body. Meanwhile, the driving unit can steer the wheel to $\beta_{i}=$ $\pm \pi / 2$. After that, when the driving unit resumes moving, the target condition is achieved. Thus, the singular target condition does not matter because the main body of SWOM can move without any problem.

In the case that the initial condition is in the singular configuration, SWOM cannot move immediately in some directions. To move in those directions, it is necessary to 
(a)

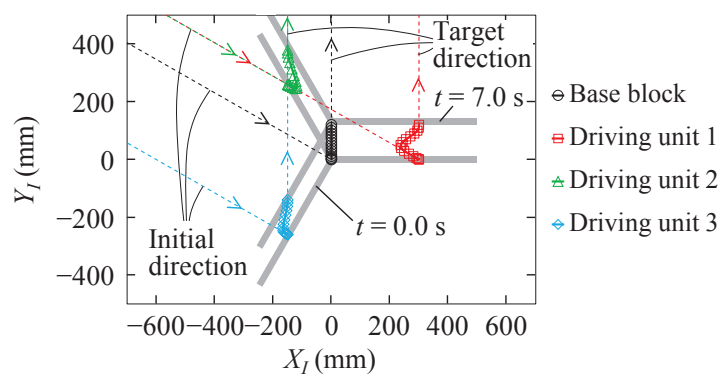

(b)

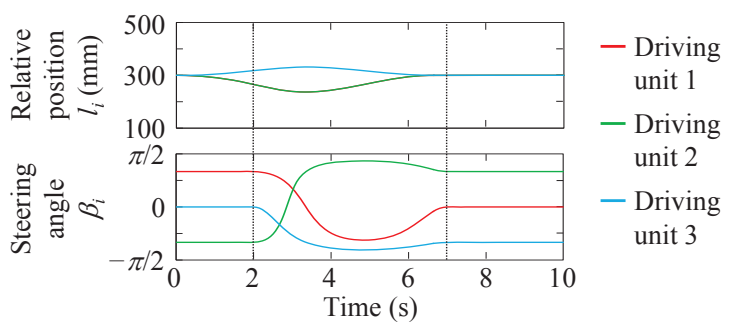

Fig. 10. Results of simulation of movement direction change in unlimited translation of SWOM. (a) Trajectories of SWOM with the colored solid lines and markers at 0.5 -s intervals during the change of the movement direction and with the colored dashed lines during unlimited translation in the initial and target conditions. (b) Graphs of the relative position of the sliding joints and the steering angles of the driving units. Note that $l_{1}=l_{2}$ occurs for the entire time.

change the direction of the wheel of the driving unit in the singular configuration from $\beta_{i}= \pm \pi / 2$. After the driving unit avoids the singular configuration, the situation is just nonsingular trajectory generation. The arrangements cost extra time to slightly steer the wheel, but it takes only a short time (e.g., $0.05 \mathrm{~s}$ ) and is not a serious problem.

Consequently, the singular configuration does not affect the movement of SWOM except in the case of the singular initial configuration. Additionally, the singular configuration, $\beta_{i}=$ $\pm \pi / 2$, is seldom required; it is required only when SWOM moves in the direction parallel to the sliding joints, i.e., $0, \pi / 3$, $2 \pi / 3, \pi, 4 \pi / 3$, and $5 \pi / 3$ from $X_{R}$. Furthermore, even if the initial condition is singular, SWOM can move by taking some preparatory time to slightly change the direction of the wheel, as mentioned above.

\section{EXPERIMENTS}

To show that SWOM can really move as described in the above sections, we conducted experiments to demonstrate SWOM movements by using the prototype.

\section{A. Experimental Method}

Fig. 11 shows the prototype of SWOM. Each sliding joint consists of two parallel linear guides. The driving unit, which has an upper part and a lower part, changes the direction of the wheel by turning the lower part relative to the upper part around a vertical axis. The upper part of the driving unit is attached to the sliders of the linear guides. Two types of motors are installed in the lower part of the driving unit: one motor turns the lower part relative to the upper part, and the other two motors rotate the wheel. Note that two driving motors are installed to increase the power, and these motors always rotate

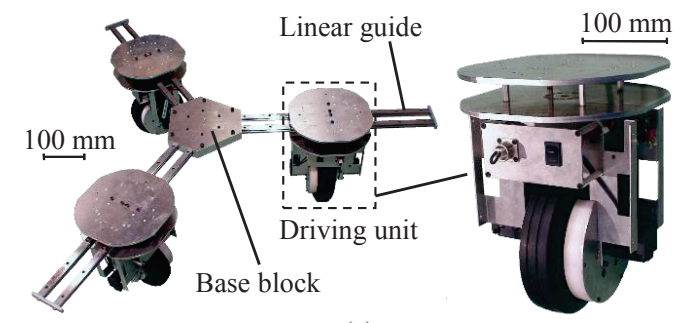

(a)

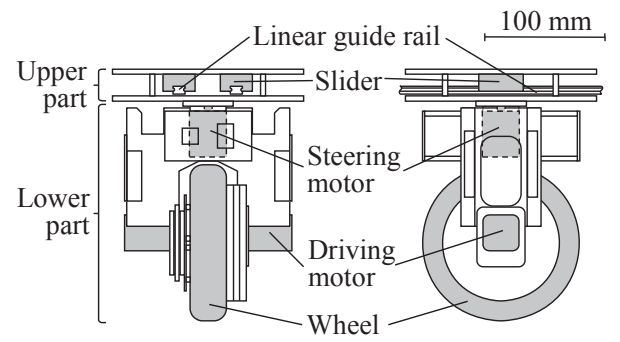

(b)

Fig. 11. (a) Prototype of SWOM. (b) Structure of the driving unit.

at the same speed, just like using one motor.

The controllers of the motors are independent of each other. The speed commands for each motor are calculated and sent in advance, and then the motors start moving at the same time when they receive a start command. This means that the prototype basically has a feedforward-control system except for each individual motor (see Fig. 15 in the Appendix). We designed this control system to make the hardware and software simple, because the purpose of the prototype is to verify the mechanism of SWOM.

We deal with three kinds of motions in the experiment. One is translation on a triangular path. The prototype moves in the right direction for $4.0 \mathrm{~s}$ at $30 \mathrm{~mm} / \mathrm{s}$, in the forward left direction for $4.0 \mathrm{~s}$ at about $42.3 \mathrm{~mm} / \mathrm{s}$, and in the backward direction for $4.0 \mathrm{~s}$ at $30 \mathrm{~mm} / \mathrm{s}$, in order. During this motion, all driving units keep the steering angles fixed at $\beta_{i}=0$. Second, the prototype moves in the right direction at $10 \mathrm{~mm} / \mathrm{s}$ while rotating clockwise at about $0.157 \mathrm{rad} / \mathrm{s}$. The steering angles of the driving units are fixed at $\beta_{i}=0$, too. These two motions show the omnidirectional mobility of SWOM under the fixed steering angles, as verified theoretically in Section III-B. Third, the unlimited translation discussed in Section IV-C is conducted. The conditions are basically the same as those in the simulation, but the prototype continues moving in the steady state until $t=10.0 \mathrm{~s}$ after finishing the change in the movement direction at $t=7.0 \mathrm{~s}$.

We measure the motion of the prototype by using the $3 \mathrm{D}$ motion tracking system VICON MX (Vicon Motion Systems Ltd) with two Bonita 10 cameras and four Bonita 3 cameras.

\section{B. Experimental Results and Discussion}

Fig. 12(a), (b), and (c) shows the experimental results of translation on a triangular path, translation with rotation, and unlimited translation, respectively. The figure shows that the measured motions generally agree with the desired motions. Thus, it is verified that the prototype of SWOM performed omnidirectional movement according to the theory derived in the previous sections and that the proposed mechanism and 


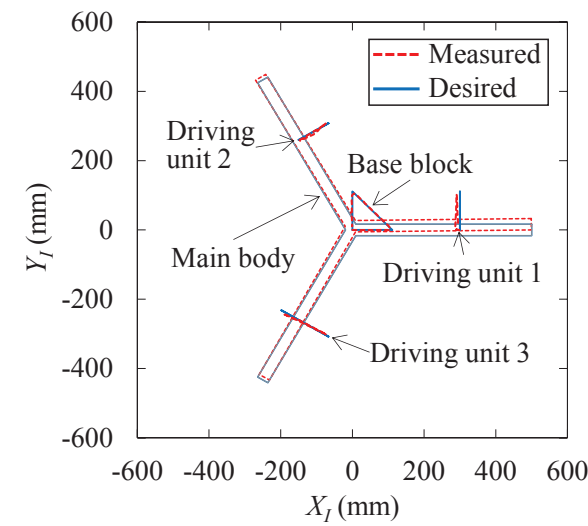

(a)

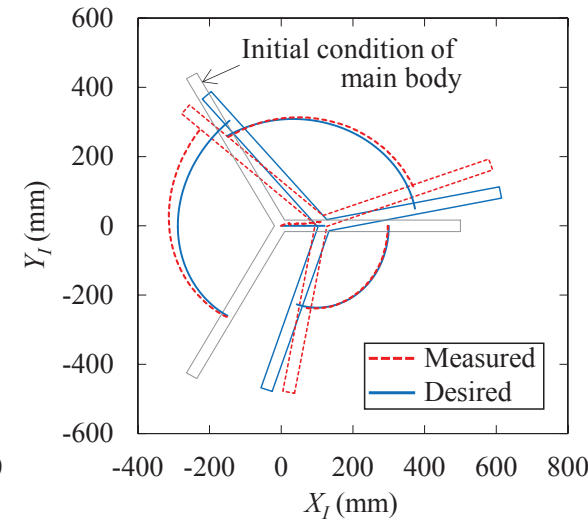

(b)

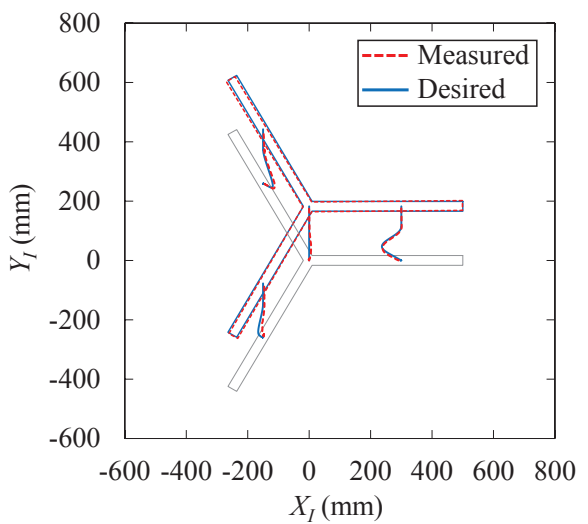

(c)

Fig. 12. Motions of SWOM prototype. (a) Translation on a triangular path. (b) Translation in $X_{I}$ direction with clockwise rotation. (c) Unlimited translation from $\chi_{0}=-\pi / 6$ to $\chi_{2}=\pi / 2$. The main body is represented by the three-forked shape. The blue solid lines are the desired paths of the driving units and the center of the base block, or the desired final condition of the main body. The red dashed lines are measured paths or the measured final condition.

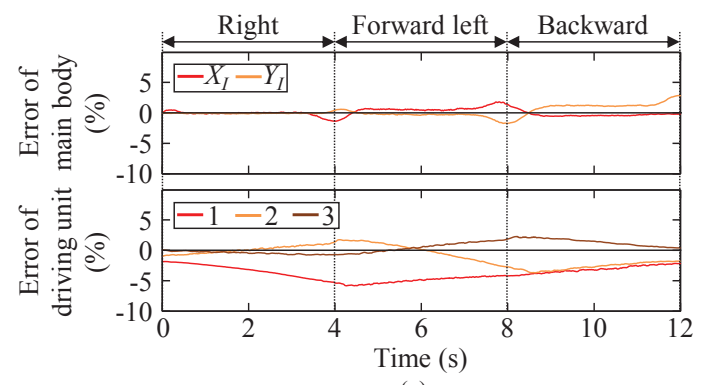

(a)

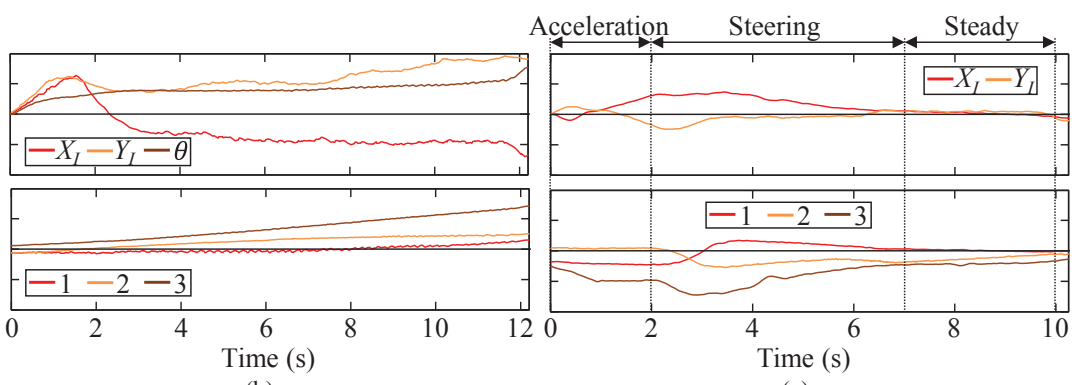

(b)

(c)

Fig. 13. Motion errors in experimental results. Upper graphs show the relative errors of the main body in the $X_{I}, Y_{I}$, and $\theta$ directions to the entire desired movement distance over time. Lower graphs shows the relative errors of driving units 1,2, or 3 in the sideways directions to each entire desired movement distance over time. (a) Result of the translation on a triangular path. (b) Result of the translation with rotation. (c) Result of the unlimited translation.

movement method are effective fundamentally.

However, by taking a closer look at the result of Fig. 12(b), the main body certainly moves in the right direction and rotates clockwise, but some motion errors occur. We analyze the errors in the results numerically. Fig. 13 shows the errors in the measured paths. The upper graphs indicate the errors of the main body in the $X_{I}, Y_{I}$, and $\theta$ [only in Fig. 13(b)] directions over time. The lower graphs indicate the errors of the driving units in the sideways directions, i.e., the directions perpendicular to each wheel plane.

In Fig. 13(a) and (c), the errors of the main body are so small that the values keep smaller than $5 \%$ for the entire time. The assumed reason is that the errors of the driving units do not always affect the errors of the main body. Actually, in Fig. 13(a), the errors of the driving units occasionally change widely and become larger than $5 \%$, especially when the movement direction of the main body is nearly parallel to the rail of the driving unit (driving unit 1 for $t=0.0-4.0 \mathrm{~s}$ and driving unit 2 for $t=4.0-8.0 \mathrm{~s}$ ). The sideways errors of the driving units cause the relative position errors on the rails. According to (11), however, the desired velocities of the driving units are independent of $l_{i}$ under the condition $\dot{\theta}=0$. Therefore, the errors of the driving units in the rail directions do not disturb the translation of the main body. By observing Fig. 13(a) and (c) in more detail, the errors of the main body become relatively large during acceleration: before and after $t=0.0,4.0,8.0,12.0 \mathrm{~s}$ in Fig. 13(a) and for $t=0.0-2.0 \mathrm{~s}$ in Fig. 13(c). The errors might be caused partly by the problem of the control system compensating for the rotation angle errors of the wheels.

In Fig. 13(b), the errors of the main body become large not only in acceleration but also after acceleration. In this case, $\dot{\theta} \neq$ 0 , the motion of the main body receives the effect of the change of $l_{i}$, and unfortunately, the errors of the driving units monotonically increase for the entire time. This is because the wheels generally tend to deviate in the centrifugal directions in rotation due to the resistance force to the turning of the wheels. The phenomenon also seems to cause the error fluctuation in Fig. 13(c) when the wheels are steered. It is difficult to predict how the wheels deviate because the wheels are affected by the friction condition or the load applied to each driving unit. However, it is thought that if sensors are installed to measure the positions of the driving units on the rails and feedback of their changes is supplied, those errors can be compensated. Especially in indoor tasks, where the friction condition does not fluctuate widely, such a feedback strategy would be effective.

Fig. 14 shows the speeds of the main body and driving units in the unlimited translation shown in Fig. 12(c). The speeds are given from the increments of the position vectors at $0.01-\mathrm{s}$ intervals. The median filter with the size of $0.10 \mathrm{~s}$ is applied to the calculated speeds. The measured speeds of the driving units basically match the desired values, but the speeds do not fully follow the desired values in large acceleration. To address this problem, we need to reconsider the performance of the motors or the control method. With respect to the speed of the main body, the errors are not so large compared to those of driving 


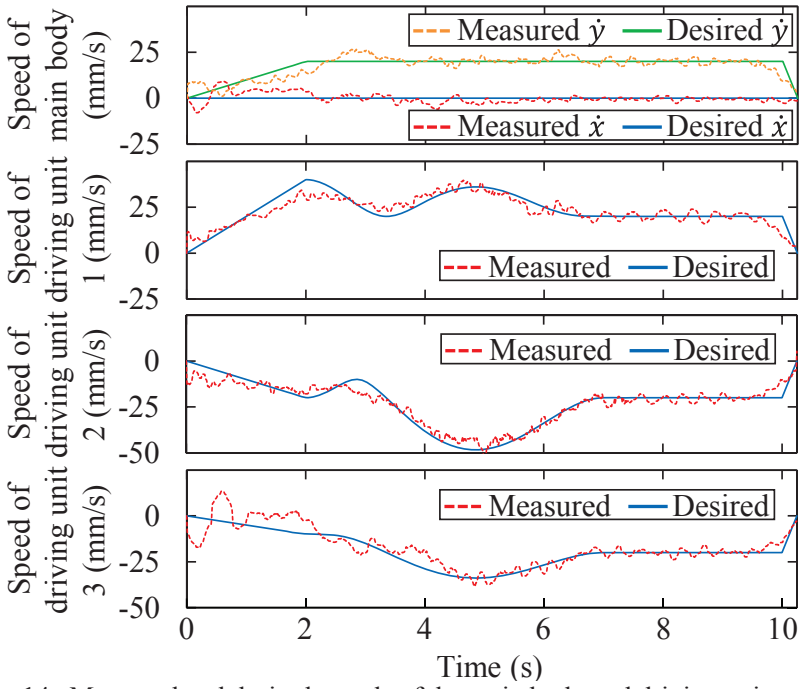

Fig. 14. Measured and desired speeds of the main body and driving units over time in the experimental result of unlimited translation.

unit 3 for $t=0.0-1.0 \mathrm{~s}$. This means that the sliding joints of SWOM do not add together the errors of the driving units in the velocities, as well as in the displacement mentioned above.

\section{CONCLUSION}

Mobile robots are used for automatically transporting products in factories and warehouses. Especially, omnidirectional mobile robots that can move immediately in an arbitrary direction have the potential for further efficiency. However, existing omnidirectional mobile robots need specialized wheel mechanisms, which can be unreliable. To solve this problem, this research proposes a novel omnidirectional mobile robot, SWOM, which has three wheels connected through sliding joints. Due to its conventional wheels without offsets between the steering axes and the ground contact points, SWOM is more reliable in structure and in motion. In this paper, we conducted analyses and experiments on SWOM and obtained the following results.

1) Through the analysis of the kinematic conditions for omnidirectional mobile robots, we proposed a novel omnidirectional mobile robot, SWOM, which uses sliding joints.

2) We derived the kinematic equations for SWOM. We proved theoretically that SWOM was able to make omnidirectional movement with respect to three-DOF planar motion, i.e., SWOM was able to move immediately in an arbitrary direction without changing the directions of the wheels.

3) We showed the reachable region of SWOM in omnidirectional movement by discussing the movable range of the sliding joints. We demonstrated that the reachable region existed in any direction of the wheels except in the singular configuration.

4) We proposed a trajectory generation method that enabled SWOM to change its movement direction while keeping the reachable region and then to continue omnidirectional movement unlimitedly in any direction.
Simulation showed that the calculated trajectories enabled SWOM to make unlimited translation in an arbitrary direction, as determined theoretically.

5) We developed a prototype of SWOM and conducted demonstrative experiments. The results verified that SWOM was able to make omnidirectional movement without steering the wheels and to achieve unlimited translation by using the proposed method.

In SWOM, unexpected movement of the passive sliding joints caused by friction and a load applied to the driving units affect the entire robot motion. To compensate such effects, we need to construct the feedback control system in future work.

\section{APPENDIX}

In Table I and Fig. 15, some details of the SWOM prototype used in the experiments are shown.

TABLE I

PROPERTIES OF SWOM PROTOTYPE

\begin{tabular}{lll} 
& \multicolumn{2}{c}{ PROPERTIES OF SWOM PROTOTYPE } \\
\hline \hline SWOM & Radius & $542 \mathrm{~mm}$ \\
& Weight & $16.4 \mathrm{~kg}$ \\
\hline Driving unit & Radius & $100 \mathrm{~mm}$ \\
& Height & $265 \mathrm{~mm}$ \\
& Weight & $3.5 \mathrm{~kg}$ \\
\hline Wheel & Radius & $78 \mathrm{~mm}$ \\
\hline Driving motor/ & Model & Futaba RS405CB \\
steering motor & Output torque & $48.0 \mathrm{kgf} \cdot \mathrm{cm}$ \\
& Rotational speed & $0.21 \mathrm{~s} / 60 \mathrm{deg}$. \\
\hline Rotary encoder & Resolution & $360 \mathrm{ppr}$ \\
\hline
\end{tabular}

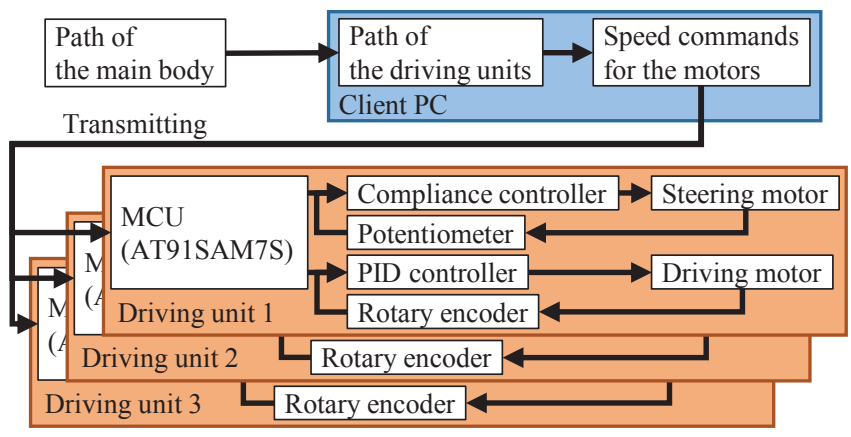

Fig. 15. Schematic diagram of the system of the SWOM prototype.

\section{ACKNOWLEDGMENT}

The authors thank H. Nishihara and T. Segoshi for their cooperation in this research. This work was supported by JSPS KAKENHI Grant Number JP18J13377.

\section{REFERENCES}

[1] P. Muir, and C. Neuman, "Kinematic modeling for feedback control of an omnidirectional wheeled mobile robot", in Proc. IEEE Int. Conf. Robot. Autom., vol.4, 1987, pp. 1772-1778.

[2] G. Campion, and G. Bastin, "On adaptive linearizing control of omnidirectional mobile robots", in Proc. Int. Symp. Math. Theory Netw. Syst., vol. 2, 1990, pp. 531-538.

[3] H. Asama, M. Sato, H. Kaetsu, K. Ozaki, A. Matsumoto, and I. Endo, "Development of an omni-directional mobile robot with 3 DOF decoupling drive mechanism", J. Robot. Soc. Jpn., vol. 14, no. 2, pp. 249-254, Mar. 1996.

[4] K. Byun, S. Kim, and J. Song, "Design of a four-wheeled omnidirectional mobile robot with variable wheel arrangement mechanism", in Proc. IEEE Int. Conf. Robot. Autom., 2002, pp. 720-725. 
[5] Y. Mei, Y. Liu, Y. Hu, and C.S. Lee, "Energy-efficient motion planning for mobile robots", in Proc. IEEE Int. Conf. Robot. Autom., 2004, pp. 4344-4349.

[6] H. Samani, A. Abdollahi, H. Ostadi, and S. Rad, "Design and development of a comprehensive omni directional soccer player robot", Int. J. Adv. Robot. Syst., vol. 1, no. 3, pp. 191-200, Sep. 2004.

[7] S. Guo, Y. Jin, S. Bao, and F.F. Xi, "Accuracy analysis of omnidirectional mobile manipulator with mecanum wheels", Advances in Manufacturing, vol. 4, no. 4, pp. 363-370, Dec. 2016.

[8] M. Komori, K. Matsuda, T. Terakawa, F. Takeoka, H. Nishihara, and H. Ohashi, "Active omni wheel capable of active motion in arbitrary direction and omnidirectional vehicle", J. Adv. Mech. Des., Syst., Manuf., vol. 10, no. 6, 2016.

[9] L. Ferrière, B. Rauccent, and G. Campoin, "Design of omnimobile robot wheels", in Proc. IEEE Int. Conf. Robot. Autom., 1996, pp. 3664-3670.

[10] F. Pin, and S. Killough, "A new family of omnidirectional and holonomic wheeled platforms for mobile robots", IEEE Trans. Robot. Autom., vol. 10, no. 4, pp. 480-489, Aug. 1994.

[11] J. Tang, K. Watanabe, and Y. Shiraishi, "Design and traveling experiment of an omnidirectional holonomic mobile robot", in Proc. IEEE/RSJ Int. Conf. Intell. Robots Syst., 1996, pp. 66-73.

[12] K. Watanabe, "Control of an omnidirectional mobile robot", in Proc. 2nd Int. Conf. Knowl. Based Intell. Electron. Syst., 1998, pp. 51-60.

[13] T. Kalmár-Nagy, P. Ganguly, and R. D'Andrea, "Real-time trajectory generation for omnidirectional vehicles", in Proc. Amer. Control Conf., 2002, pp. 286-291.

[14] X. Wang, W. Cui, X. Xu, and C. Ye, "Research on an omni-directional AGV with differential wheels", in Proc. IEEE Int. Conf. Mechatronics Autom., 2016, pp. 1566-1571.

[15] M. West, and H. Asada, "Design and control of ball wheel omnidirectional vehicles", in Proc. IEEE Int. Conf. Robot. Autom., 1995, pp. 1931-1938.

[16] S. Ostrovskaya, R.J. Spiteri, and J. Angeles, "Dynamics of a mobile robot with three ball-wheels", Int. J. Robot. Res., vol. 19, no. 4, pp. 383-393, Apr. 2000.

[17] M. Wada, and H. Asada, "Design and control of a variable footprint mechanism for holonomic omnidirectional vehicles and its application to wheelchairs", IEEE Trans. Robot. Autom., vol. 15, no. 6, pp. 978-989, Dec. 1999.

[18] S. Ok, A. Kodama, Y. Matsumura, and Y. Nakamura, " $\mathrm{SO}(2)$ and $\mathrm{SO}(3)$, omni-directional personal mobility with link-driven spherical wheels", in Proc. IEEE/RSJ Int. Conf. Intell. Robots Syst., 2011, pp. 268-273.

[19] W. Chen, C. Chen, J. Tsai, J. Yang, and P. Lin, "Design and implementation of a ball-driven omnidirectional spherical robot", Mech. Mach. Theory, vol. 68, pp. 35-48, Oct. 2013.

[20] A. Singh, A. Paigwar, S.T. Manchukanti, M. Saroya, M. Maurya, and S. Chiddarwar, "Design and implementation of Omni-directional spherical modular snake robot (OSMOS)", in Proc. Int. Conf. Mechatronics, 2017, pp. $79-84$.

[21] O. Khatib, K. Yokoi, K. Chang, D. Ruspini, R. Holmberg, and A. Casal, "Coordination and decentralized cooperation of multiple mobile manipulators", J. Robot. Syst., vol. 13, no. 11, pp. 755-764, Nov. 1996.

[22] O. Khatib, "Mobile manipulation: the robotic assistant", Robot. Auton. Syst., vol. 26, no. 2-3, pp. 175-183, Feb. 1999.

[23] R. Holmberg, and O. Khatib, "Development and control of a holonomic mobile robot for mobile manipulation tasks", Int. J. Robot. Res., vol. 19, no. 11 , pp. 1066-1074, Nov. 2000.

[24] M. Wada, and S. Mori, "Development of a holonomic and omnidirectional mobile robot", J. Robot. Soc. Jpn., vol. 15, no. 8, pp. 1139-1146, Nov. 1997.

[25] M. Wada, A. Takagi, and S. Mori, "A mobile platform with a dual-wheel caster-drive mechanism for holonomic and omnidirectional mobile robots", J. Robot. Soc. Jpn., vol. 18, no. 8, pp. 1166-1172, Nov. 2000.

[26] M. Wada, "Design and analysis of a wheeled platform with a synchro caster-drive mechanism for holonomic and omnidirectional mobile robots", J. Robot. Soc. Jpn., vol. 19, no. 6, pp. 784-792, Sep. 2001.

[27] A. El-Shenawy, A. Wagner, and E. Badreddin, "Controlling a holonomic mobile robot with singularities", in Proc. World Congr. Intell. Control Autom., 2006, pp. 8270-8274.

[28] J. Ma, H. Kharboutly, A. Benali, F.B. Amar, and M. Bouzit, "Design of omnidirectional mobile platform for balance analysis", IEEE/ASME Trans. Mechatronics, vol. 19, no. 6, pp. 1872-1881, Dec. 2014.

[29] E.J. Jung, B.J. Yi, and W. Kim, "Kinematic analysis and motion planning for a planar multiarticulated omnidirectional mobile robot", IEEE/ASME Trans. Mechatronics, vol. 20, no. 6, pp. 2983-2995, Oct. 2015.
[30] G. Campoin, G. Bastin, and B. D'Andréa-Novel, "Structural properties and classification of kinematic and dynamic models of wheeled mobile robots", IEEE Trans. Robot. Autom., vol. 12, no. 1, pp. 47-62, Feb. 1996.

[31] R. Siegwart, I.R. Nourbakhsh, and D. Scaramuzza, Introduction to Autonomous Mobile Robots, 2nd ed., Cambridge, MA: The MIT Press, 2011, pp. 63-71.

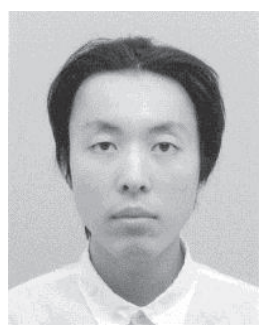

Tatsuro Terakawa received the B.E. degree in mechanical engineering from Kyoto University, Japan, in 2014, and the M.E. degree from Kyoto University Graduate School of Engineering, Department of Mechanical Engineering and Science, in 2016, where he is currently working toward the $\mathrm{Ph} . \mathrm{D}$. degree in engineering.

His research in Kyoto University is focused on mobile robots, actuator mechanisms, and design.

Mr. Terakawa received JSME Miura award, the JSME MD\&T division encouragement presentation, and JSAE graduate school research encouragement award in 2016.

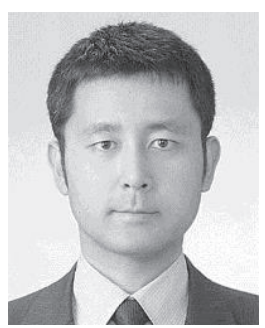

Masaharu Komori (M'16) received the B.E. degree in precision engineering from Kyoto University in 1995 and the M.E. degree from the Graduate School of Kyoto University in 1997. From 1999 to 2000, he was a student in the doctoral course in Kyoto University and, in 2000, he became a research assistant in the Graduate School of Engineering of Kyoto University. He received the doctor's degree in engineering from Kyoto University in 2002.

In 2004, he became an associate professor in Kyoto University and now belongs to the Department of Mechanical Engineering and Science at Kyoto University. His research interest includes robots, transmission, laser interferometric measurements, artifact design, gear checker analysis and evaluation, performance analysis of gears, surface finishing methods, and micro/nanoimprinting.

Dr. Komori is member of the RSJ (Robotics Society of Japan), JSME (Japan Society of Mechanical Engineers), JSDE (Japan Society of Design Engineering), and JSPE (Japan Society for Precision Engineering). He was a recipient of The Young Scientists' Prize of The Commendation for Science and Technology by the Minister of Education, Culture, Sports, Science and Technology in 2011, a WT award in 2013, two JSME medals for outstanding paper in 2009 and 2013, a JSME medal for new technology in 2014, a JSME young engineers award in 2005, a best paper award from the JSDE in 2012, and an Eiji Mutoh excellent design award in 2012. His paper was selected as one of the best articles published in Measurement Science and Technology during 2009.

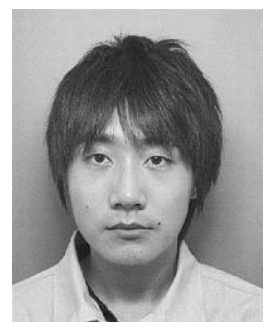

Kippei Matsuda received the B.E. and M.E. degrees in mechanical engineering from Kyoto University, Japan, in 2008 and 2010, respectively.

His research in Kyoto University was on mobile robots.

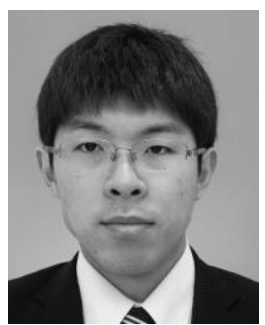

Shinji Mikami received the B.E. degree in mechanical engineering from Kyoto University, Japan, in 2016.

His research in Kyoto University was on mobile robots. 